\title{
Primer registro de Vigna vexillata (Fabaceae, Faboideae) en Sinaloa, México
}

\section{First record of Vigna vexillata (Fabaceae, Faboideae) for Sinaloa, Mexico}

\author{
Francisco Amador-Cruz 1,3 (D), Daniel Benítez-Pardo' (D), Raquel Briseño-Dueñas² (D)
}

1 Universidad Autónoma de Sinaloa, Facultad de Ciencias del Mar, Laboratorio de Botánica Acuática, Paseo Claussen s.n., Col. Los pinos, Apdo. postal 61082000 Mazatlán, Sinaloa, México.

2 Universidad Nacional Autónoma de México, Instituto de Ciencias del Mar y Limnología, Unidad Académica Mazatlán, Av. Joel Montes Camarena s.n. Col. Cerro del Vigía, 82040 Mazatlán, Sinaloa, México.

3 Autor para la correspondencia: famadorc.facimar@uas.edu.mx; facbio2000@hotmail.com

\section{Citar como:}

Amador-Cruz, F., D. Benítez-Pardo y R. Briseño-Dueñas. 2017. Primer registro de Vigna vexillata (Fabaceae, Faboideae) en Sinaloa, México. Acta Botanica Mexicana 121: 169-176. DOI: http://dx.doi. org/10.21829/abml21.2017.1243

Recibido: 18 de abril de 2017

Revisado: 16 de mayo de 2017.

Aceptado: 23 de junio de 2017

DOI:

http://dx.doi.org/10.21829/abml21.2017.1243

\section{Resumen:}

Antecedentes y Objetivos: La Flora de Sinaloa está compuesta por 3736 especies registradas; sin embargo, su conocimiento es relativamente escaso y la mayoría de los estudios florísticos se han realizado en la porción norte, por lo cual se desarrolló en la zona sur el presente trabajo. Éste tiene como objetivo contribuir al conocimiento de dicha flora, a través de un nuevo registro para la especie Vigna vexillata, a nivel estatal.

Métodos: Se realizaron cinco salidas a lo largo de la zona de estudio, durante las cuales se recolectaron plantas, siguiendo las recomendaciones para la preparación de ejemplares botánicos; estas se determinaron con ayuda de bibliografía taxonómica especializada.

Resultados clave: Se encontró un taxon del género Vigna que no estaba reportado para la flora del estado de Sinaloa. Se reconoce por presentar el giro de quilla hacia la izquierda; las estípulas foliales cordadas; un fruto terete lineal y la pubescencia marrón. La especie fue colectada en la zona costera de Escuinapa. El registro extiende la distribución de la especie a una zona más norteña.

Conclusiones: Este registro sugiere la necesidad de continuar con los trabajos de flora en la zona sur de Sinaloa, ya que es un área escasamente estudiada y que presenta una gran variedad de tipos de vegetación, los cuales han permanecido inexplorados.

Palabras clave: florística, norte de México, sur de Sinaloa, taxonomía.

\section{ABSTRACT:}

Background and Aims: The Flora of Sinaloa consists of 3736 species; however, the knowledge of it is scarce. Most of the studies have been carried out in the northern portion, whereas the present work was developed in the south zone. The objective is to contribute to the knowledge of this flora, through a new record of the species Vigna vexillata at state level.

Methods: Throughout the study area, five field trips were carried out, during which plants were collected, following the recommendations for the preparation of botanical specimens; these were determined with specialized taxonomic bibliography.

Key results: A taxon of the genus Vigna was determined, which to date was not reported for the flora of Sinaloa. It is recognized for presenting the keel turn to left, cordate stipules, terete and linear fruit, and brown pubescence. The species was collected in the Escuinapa coastal area. This record extends the distribution of the species to a more northern area.

Conclusions: This record suggests the need to continue observing the flora in the south area of the state of Sinaloa, since it is scarcely studied and presents a great variety of types of vegetation, which have remained unexplored.

Key words: floristics, north Mexico, south Sinaloa, taxonomy. 


\section{INTRODUCCIÓN}

La flora del estado de Sinaloa ha sido escasamente estudiada a pesar de albergar una gran riqueza vegetal debido a que se encuentra en una zona de confluencia de dos provincias florísticas: la Costa Pacífica y la Planicie Costera del Noroeste (Vega-Aviña, 2000; Rzedowski, 2006). No se tiene una estimación real del número de especies en el estado; se tenían contempladas 3500 (Vega-Aviña, 2000), pero Villaseñor-Ríos y Ortiz-Bermúdez (2014) mencionan 2922, y los estudios más recientes de Villaseñor-Ríos (2016) establecen 3736, de las cuales 69 son endémicas de México y dos tienen distribución restringida al estado.

La mayoría de los trabajos botánicos se han enfocado a la porción norte de Sinaloa: Brandegee $(1905,1906)$; Ponce de León (1909); Riley (1923a, b, c, d, 1924); González-Ortega (1929); Shreve (1934, 1937); Gentry (1946a, b, 1948); Vega-Aviña (2000); Vega-Aviña et al. (2000, 2008) y Saturnino-Díaz (2008). De la zona sur sólo se tienen reportados los estudios de Flores-Campaña et al. (1996) y Van Devender et al. (2012), los cuales abarcan los municipios Concordia y Mazatlán.

Los trabajos mencionados reportan al menos una especie del género Vigna Savi; en total se tienen registradas tres para Sinaloa: $V$. adenantha (G. Mey.) Maréchal, Mascherpa \& Stainier, V. speciosa (Kunth) Verdc. y $V$. peduncularis Fawc. \& Rendle. El género Vigna es pantropical (Delgado-Salinas et al., 2011) e incluye cerca de 100 especies, distribuidas en siete subgéneros (Aitawade et al., 2012). Los subgéneros Plectotropis y Ceratotropis se caracterizan por presentar un giro en la quilla hacia la izquierda; dentro del primero encontramos a la especie Vigna vexillata (L.) A. Rich. (Karuniawan et al., 2006; Delgado-Salinas et al., 2011).

El objetivo del presente trabajo es registrar y describir a Vigna vexillata para el estado de Sinaloa, así como presentar la distribución del taxon y de esta manera contribuir al conocimiento de la flora estatal.

\section{Materiales y Métodos}

La región Palmito del Verde se ubica en la zona sur del estado de Sinaloa, la mayoría de su superficie pertenece al mu- nicipio de Escuinapa y una pequeña parte al municipio de Rosario, se encuentra entre las coordenadas $22^{\circ} 49^{\prime} 47.2^{\prime \prime} \mathrm{N}$, $106^{\circ} 01^{\prime} 13.2^{\prime \prime O}$ y $22^{\circ} 28^{\prime} 32.98^{\prime \prime} \mathrm{N}, 105^{\circ} 40^{\prime} 51.6^{\prime \prime O}$ (Fig. 1).

A lo largo de la zona de estudio se realizaron cinco salidas de campo (de enero a marzo de 2017), en las cuales se recorrieron diferentes rutas para poder obtener un inventario florístico; las muestras botánicas fueron colectadas siguiendo la metodología propuesta por Lot y Chiang (1986). Posteriormente fueron llevados al Laboratorio de Botánica Acuática de la Facultad de Ciencias del Mar de la Universidad Autónoma de Sinaloa, donde se determinaron con la bibliografía taxonómica especializada de McVaugh (1987), Beyra y Reyes-Artiles (2004) y Delin y Thulin (2010). Asimismo, se corroboró su identidad con especialistas del género, y finalmente se depositaron en el herbario Jesús González Ortega (UAS), en Culiacán, Sinaloa, de la Universidad Autónoma de Sinaloa.

\section{Resultados}

Durante el proceso de determinación de los especímenes colectados para el proyecto "Caracterización de la vegetación y composición florística del sur de Sinaloa, con fines de manejo y conservación", realizados en 2017, se encontraron ejemplares del género Vigna, distintos a $V$. adenantha, $V$. speciosa y $V$. peduncularis, especies conocidas para el estado (Brandegee, 1905, 1906; Ponce de León, 1909; Riley 1923a, b, c, d, 1924; González-Ortega, 1929; Shreve, 1934, 1937; Gentry, 1946a, b, 1948; McVaugh, 1987; Flores-Campaña et al., 1996; Vega-Aviña, 2000; Vega-Aviña et al., 2000; Saturnino-Díaz, 2008; Vega-Aviña et al., 2008; Van Devender et al., 2012; Villaseñor-Ríos, 2016). La identidad de las muestras botánicas corresponde a $V$. vexilla$t a$, la cual constituye un nuevo registro para la entidad. A pesar de ser una especie morfológicamente variable, se puede determinar fácilmente debido a que la quilla tiene un giro hacia la izquierda, las estípulas foliales son cordadas, y el fruto es terete, linear, alrededor de $10 \mathrm{~cm}$ de largo, 4-5 mm de diámetro y presenta pubescencia marrón (Hedström y Thulin, 1986; McVaugh, 1987; Karuniawan et al., 2006). 


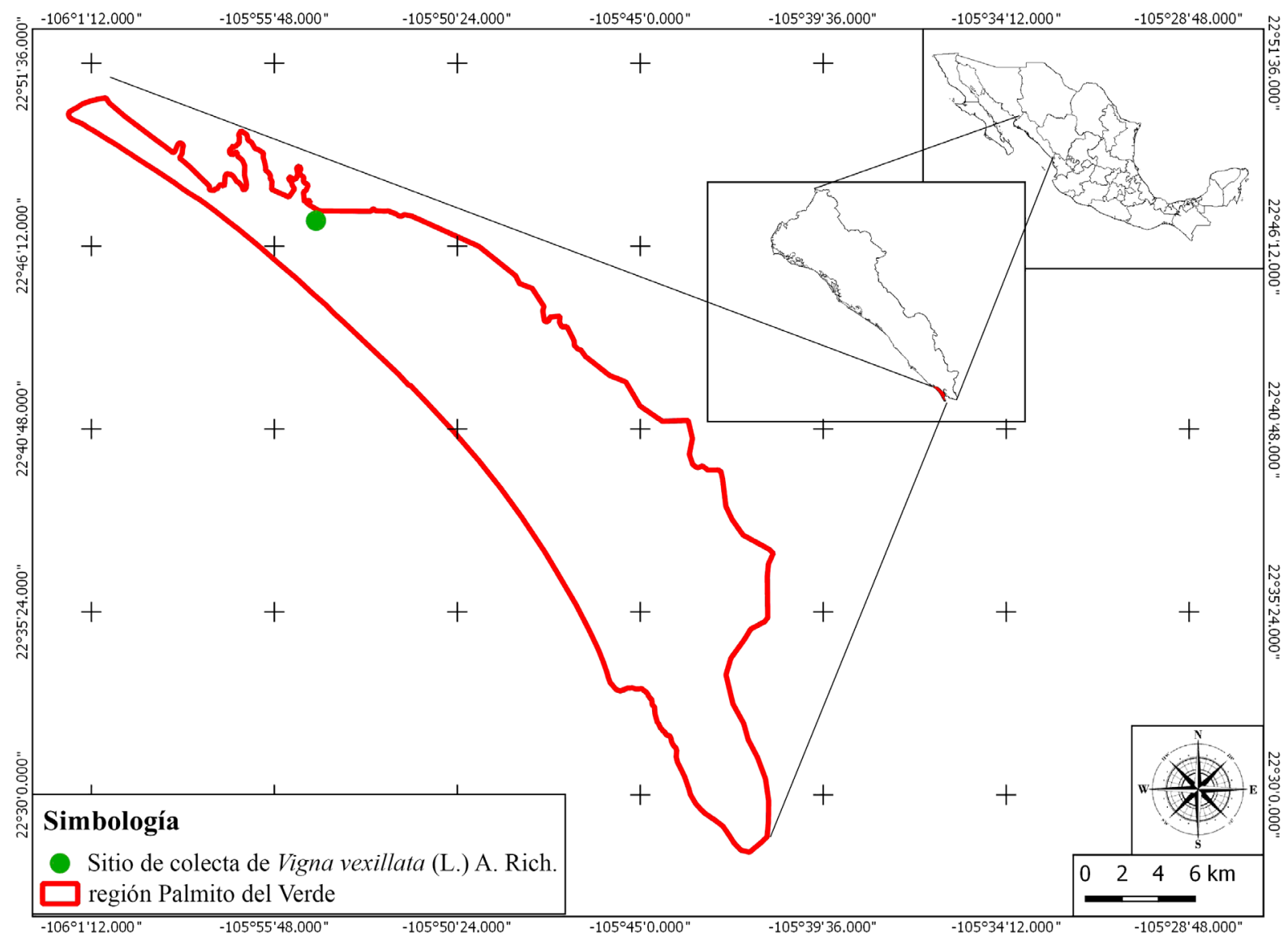

Figura 1: Distribución geográfica de Vigna vexillata (L.) A. Rich., dentro de la región Palmito del Verde, Sinaloa, México. Francisco Amador-Cruz.

La especie se localizó a dos metros de un cuerpo de agua, cuya profundidad varía según la época del año y se encuentra en la zona de conservación del proyecto Centro Integralmente Planeado y Sustentable (CIP), Playa Espíritu, Escuinapa, Sinaloa, del Fondo Nacional de Fomento al Turismo (FONATUR), en las coordenadas $22^{\circ} 46^{\prime} 52.4^{\prime \prime} \mathrm{N}, 105^{\circ} 54^{\prime} 36.2$ "O (Fig. 1).

Los individuos observados presentaron flor y fruto en los meses de febrero y marzo. El medio donde habita la planta está rodeado por vegetación secundaria de selva baja caducifolia, con una dominancia de Acacia pennatula (Schltdl. \& Cham.) Benth. y Prosopis juliflora (Sw.) DC., así como elementos de vegetación acuática (Typha domingensis Pers. y Cyperus sp.). La especie utilizaba de soporte a individuos de Tephrosia cinerea Pers., Cyperus sp. y Acacia pennatula.
Ejemplares examinados: MÉXICO. Sinaloa, municipio Escuinapa, Las Cabras; 22 46'52.4"N, 105'54'36.2"O, 6.II.2017, Amador-Cruz 26 (UAS), 27 (UAS), 28 (UAS), 29 (UAS) (Figs. 2, 3).

\section{DISCUSIÓN}

La especie Vigna vexillata se desarrolla en los trópicos y subtrópicos de ambos hemisferios, teniendo dos centros de diversificación: África y Asia (Beyra y Reyes-Artiles, 2004; Karuniawan et al., 2006). De manera particular en América, a pesar de ser una especie introducida, se considera naturalizada, desarrollándose en ambientes de vegetación secundaria de selva baja caducifolia o selva mediana subcaducifolia. Su mecanismo de dispersión es mediante legumbres espermobólicas, las cuales son bas- 

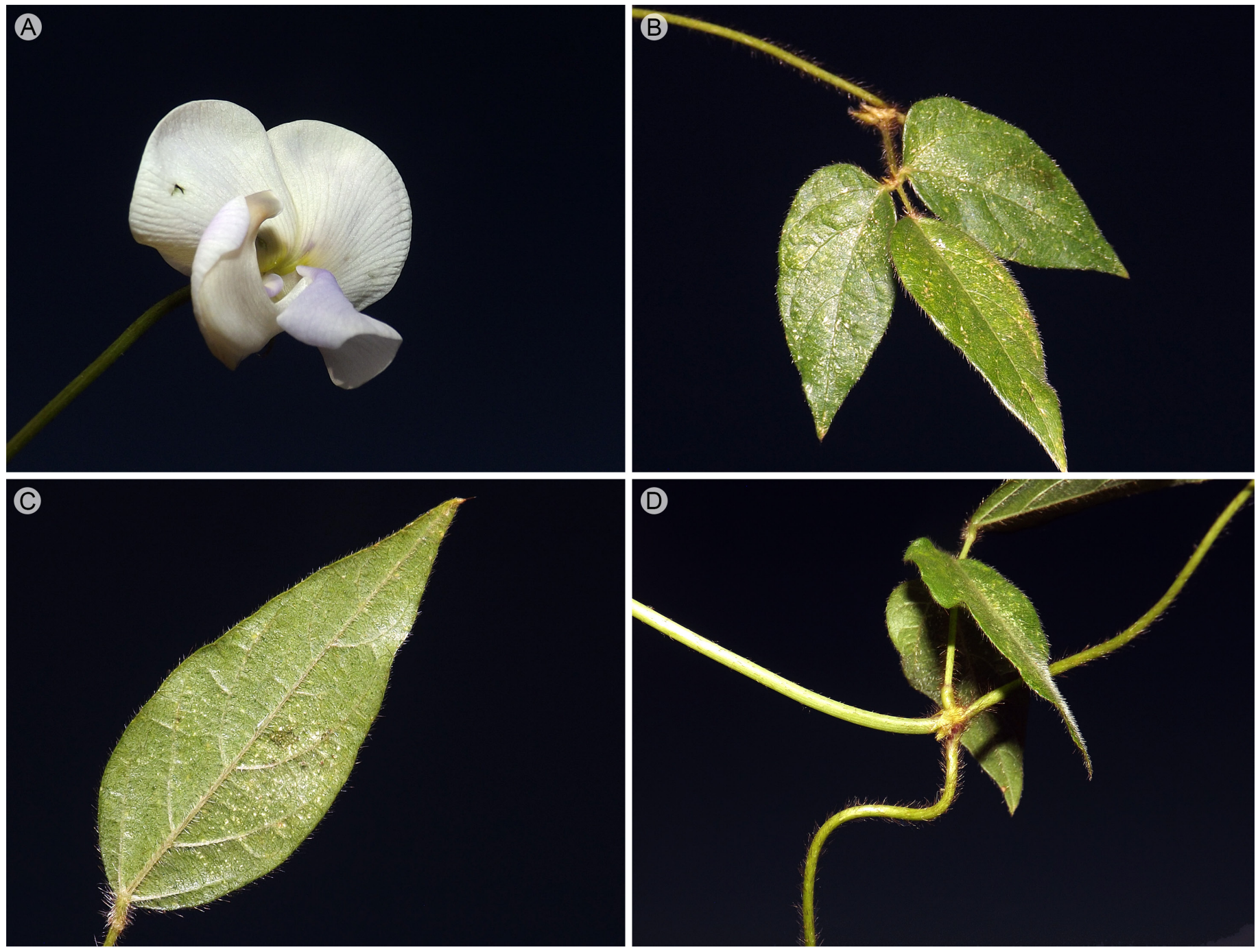

Figura 2: Vigna vexillata (L.) A. Rich. A. flor de la planta en su hábitat; B. hoja trifoliolada con borde entero y abundante pubescencia; C. detalle de un foliolo por el envés; D. detalles del tallo y punto de inserción de la hoja. Fotografías: Francisco Amador-Cruz.

tante eficientes (Díaz-Suárez y Ríos-Albuerne, 2017) y aseguran la dispersión a cierta distancia de la planta madre (Wilson, 1981). Se reportan diferentes usos para la especie como el medicinal, forrajero, control de erosión y comestible (raíz) (Beyra y Reyes-Artiles, 2004; GRINGLOBAL, 2006; Karuniawan et al., 2006).

En México se tienen reportes de la especie para Campeche, Chiapas, Guerrero, Hidalgo, Jalisco, Michoacán, Morelos, Nayarit, Oaxaca, Quintana Roo, Tabasco, Tamaulipas, Veracruz y Yucatán. Con este nuevo registro el estado de Sinaloa se considera como el límite más septentrional de la costa occidental mexicana (McVaugh, 1987; Vibrans-Lindemann, 2011; Villaseñor-Ríos, 2016; TROPICOS, 2017).

\section{CONCLUSIONES}

Este registro sugiere la necesidad de continuar con los estudios de flora en la zona sur de Sinaloa, ya que es un área escasamente estudiada y que presenta una gran variedad de tipos de vegetación, los cuales han permanecido inexplorados hasta hace poco tiempo, debido al difícil acceso. El conocimiento generado se podría encaminar al aprovechamiento de esos recursos. 


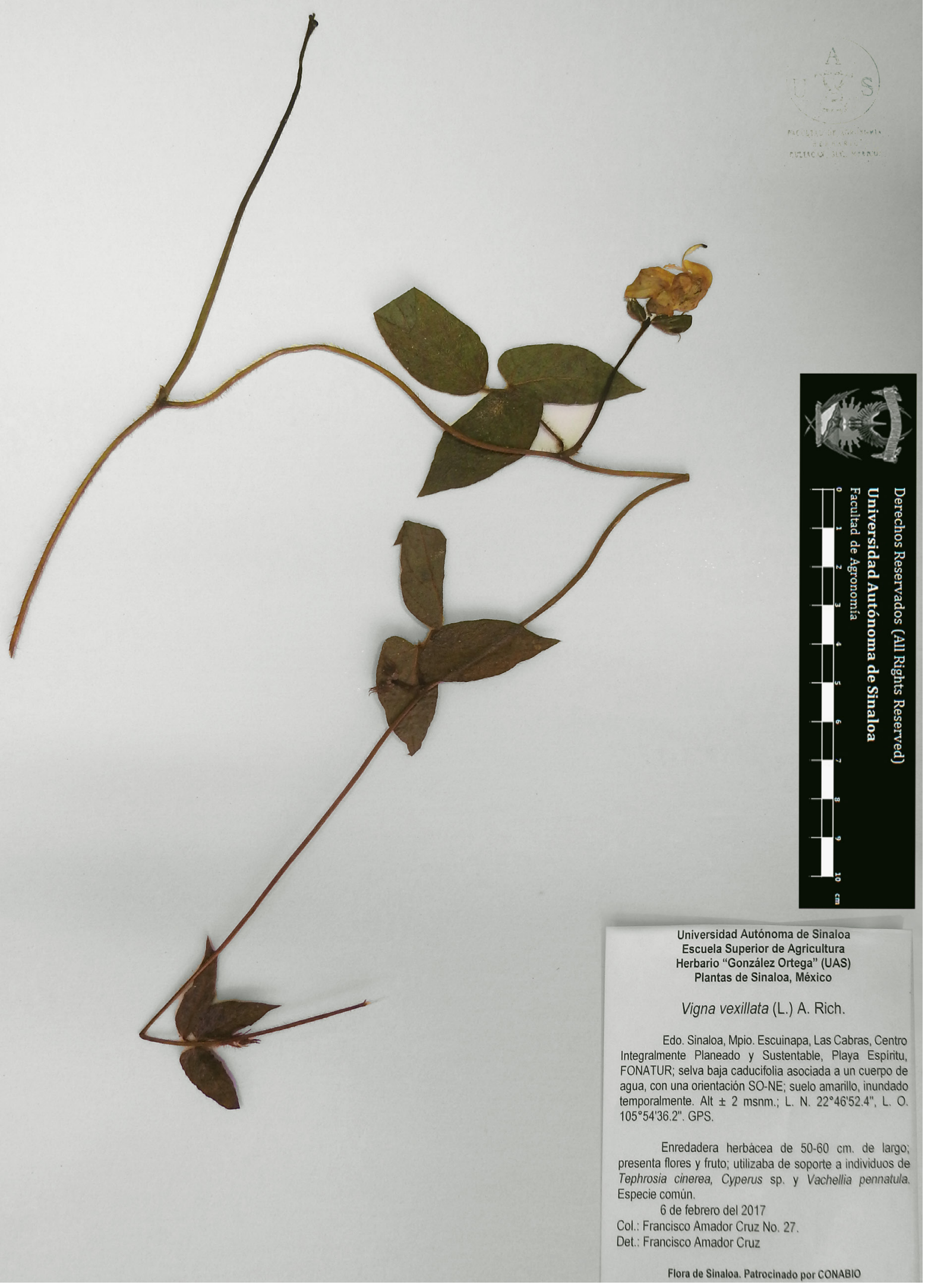

Figura 3: Vigna vexillata. Ejemplar herborizado y almacenado en el herbario Jesús González Ortega (UAS). Fotografía: Francisco Amador-Cruz. 


\section{CONTRIBUCIONES DE AUTORES}

FAC y DBP llevaron a cabo las recolectas de ejemplares botánicos. FAC realizó la determinación de los ejemplares, la toma de fotografías y la revisión de los estudios de referencia. RBD fue responsable de obtener los permisos necesarios para ingresar a las instalaciones del CIP del FONATUR. FAC escribió el documento con el apoyo de DBP y RBD. Todos los autores contribuyeron a la discusión, revisión y aprobación del manuscrito.

\section{FINANCIAMIENTO}

Este estudio fue apoyado por el Programa de Fomento y Apoyo a Proyectos de Investigación (PROFAPI) otorgado por la Universidad Autónoma de Sinaloa (número de proyecto PROFAPI2015/039) a DBP, y por el Consejo Nacional de Ciencia y Tecnología (número de beca 769304) a FAC.

\section{AgRADECIMIENTOS}

Al programa de Maestría en Ciencias en Recursos Acuáticos, de la Facultad de Ciencias del Mar, de la Universidad Autónoma de Sinaloa; al Consejo Nacional de Ciencia y Tecnología (beca 769304); a Josué de la Cruz Esparza, Julieta Álvarez Servín y Edith Flores Wence por el apoyo en el trabajo de campo; a Alfonso Delgado Salinas, Rito Vega Aviña y Heike Vibrans Lindemann por el apoyo en la determinación de la especie; a María Isabel Ramírez Ochoa por sus comentarios y sugerencias para enriquecer el manuscrito; al FONATUR por el acceso a sus instalaciones y a los revisores anónimos por sus valiosos comentarios y sugerencias.

\section{LITERATURA CITADA}

Aitawade, M. M., S. P. Sutar, S. R. Rao, S. K. Malik, S. R. Yadav y K. V. Bhat. 2012. Section Ceratotropis of subgenus Ceratotropis of Vigna (Leguminosae-Papilionoideae) in India with a new species from northern Western Ghats. Rheedea 22(1): 20-27.

Arriaga-Cabrera, L., V. Aguilar-Sierra y J. M. Espinoza. 2009. Regiones prioritarias y planeación para la conservación de la biodiversidad. In: Dirzo, R., R. Gónzalez e I. J. March (comps.). 2009. Capital natural de México, Vol. II: Estado de conservación y tendencias de cambio. Comisión
Nacional para el Conocimiento y Uso de la Biodiversidad. México, D.F., México. Pp. 433-457.

Beyra, A. y G. Reyes-Artiles. 2004. Revisión taxonómica de los géneros Phaseolus y Vigna (LeguminosaePapilionoideae) en Cuba. Anales del Jardín Botánico de Madrid 61(2): 135-154. DOI: http://dx.doi.org/10.3989/ ajbm.2004.v61.i2.41

Brandegee, T. S. 1905. Plants from Sinaloa, Mexico. Zoe 5(10): 196-226.

Brandegee, T. S. 1906. Plants from Sinaloa, Mexico. Zoe 5(11): 241-246.

Delgado-Salinas, A., M. Thulin, R. Pasquet, N. Weeden y M. Lavin. 2011. Vigna (Leguminosae) sensu lato: the names and identities of the american segregate genera. American Journal of Botany 98(10): 1694-1715.

Delin, W. y M. Thulin. 2010. Vigna. Flora de China. URL: http://flora.huh.harvard.edu/china/mss/volume 10/ FOC_10_Fabaceae_all.pdf/(consultado febrero de 2017).

Díaz-Suárez, L. y C. Ríos-Albuerne. 2017. Diásporas de las arvenses más agresivas en los agroecosistemas de Cuba. Revista Centro Agrícola 44(2): 75-82.

Flores-Campaña, L. M., R. Vega-Aviña，D. Benítez-Pardo y F. Hernández-Álvarez. 1996. Flora de Isla Venados, bahía de Mazatlán, Sinaloa, México. Anales Instituto de Biología, Universidad Nacional Autónoma de México, Serie Botánica 67(2): 283-301.

Gentry, H. S. 1946a. Sierra Tacuichamona-a Sinaloa plant locale. Bulletin of the Torrey Botanical Club 73(4): 356362. DOI: http://dx.doi.org/10.2307/2481546

Gentry, H. S. 1946b. Notes on the vegetation of Sierra Surotato in Northern Sinaloa. Bulletin of the Torrey Botanical Club 73(5): 451-462. DOI: http://dx.doi.org/10.2307/2481592

Gentry, H. S. 1948. Additions to the flora of Sinaloa and Nuevo Leon. Brittonia 6(3): 309-331. DOI: http://dx.doi. org/10.2307/2804834

González-Ortega, J. 1929. Flora indígena de Sinaloa. Boletín de pro-cultura regional, Mazatlán 1(1): 1-12.

GRIN-GLOBAL. 2006. Vigna vexillata (L.) A. Rich. U.S. National Plant Germplasm System. URL: https:// npgsweb.ars-grin.gov/gringlobal/taxonomydetail. aspx?41649 (consultado febrero de 2017). 
Hedström, I. y M. Thulin. 1986. Pollination by a hugging mechanism in Vigna vexillata (LeguminosaePapilionoideae). Plant Systematics and Evolution 154(3): 275-283. DOI: http://dx.doi.org/10.1007/BF00990129

Karuniawan, A., A. Iswandi, P. R. Kale, J. Heinzemann y W. J. Grüneberg. 2006. Vigna vexillata (L.) A. Rich. cultivated as a root crop in Bali and Timor. Genetic Resources and Crop Evolution 53(1): 213-217. DOI: http://dx.doi. org/10.1007/s10722-005-1654-5

Lot, A. y F. Chiang (comps.). 1986. Manual de Herbario. Administración y manejo de colecciones, técnicas de recolección y preparación de ejemplares botánicos. Consejo Nacional de la Flora de México, A.C. México, D.F., México. 142 pp.

McVaugh, R. 1987. Leguminosae. Flora Novo-Galiciana 5: $1-786$.

Ponce de León, R. 1909. Ligeros apuntes sobre la flora del Estado de Sinaloa. Talleres Tipográficos de Julio G. Arce “Rosales” Núm. 24. Culiacán, México. 33 pp.

Riley, L. A. M. 1923a. Contributions to the Flora of Sinaloa: I. Bulletin of Miscellaneous Information (Kew) 1923(3): 103-115. DOI: http//dx.doi.org/10.2307/4118296

Riley, L. A. M. 1923b. Contributions to the Flora of Sinaloa: II. Bulletin of Miscellaneous Information (Kew) 1923(4): 163-175. DOI: http://dx.doi.org/10.2307/4118538

Riley, L. A. M. 1923c. Contributions to the Flora of Sinaloa: III. Bulletin of Miscellaneous Information (Kew) 1923(9): 333-346. DOI: http://dx.doi.org/10.2307/4120239

Riley, L. A. M. 1923d. Contributions to the Flora of Sinaloa: IV. Bulletin of Miscellaneous Information (Kew) 1923(10): 388-401. DOI: http://dx.doi.org/10.2307/4115420

Riley, L. A. M. 1924. Contributions to the Flora of Sinaloa: V. Bulletin of Miscellaneous Information (Kew) 1924(5): 206-222. DOI: http://dx.doi.org/10.2307/4111748

Rzedowski, J. 2006. Vegetación de México. 1ra. ed. digital. Comisión Nacional para el Conocimiento y Uso de la Biodiversidad. México, D.F., México. 504 pp.

Saturnino-Díaz, J. 2008. Diversidad florística y estructura de la vegetación de las islas de los sistemas lagunares Navachiste y Macapule, del norte de Sinaloa. Tesis de maestría. Instituto Politécnico Nacional-Centro
Interdisciplinario de Investigación para el Desarrollo Integral Regional. Guasave, México. 133 pp.

Shreve, F. 1934. Vegetation of the Northwestern Coast of Mexico. Bulletin of the Torrey Botanical Club 61(7): 373 380. DOI: http://dx.doi.org/10.2307/2481022

Shreve, F. 1937. Lowland vegetation of Sinaloa. The Bulletin of the Torrey Botanical Club 64(9): 605-613. DOI: http:// dx.doi.org/10.2307/2481132

TROPICOS. 2017. Tropicos.org. Missouri Botanical Garden. URL: http://tropicos.org/Home.aspx (consultado marzo de 2017).

Van Devender, T. R., M. Ruiz Guerrero, A. L. Reina Guerrero, P. Mejía Mora, S. Guido Sánchez y A. M. van der Heiden. 2012. Flora de La Guásima, Concordia, Sinaloa, México. Región Prioritaria para la Conservación. Versión 1.0 (agosto 2012). 49 pp. URL: http://www.ciad.mx/herbario (consultado febrero de 2017).

Vega-Aviña, R. 2000. Catálogo y base de datos preliminar de la flora de Sinaloa. Universidad Autónoma de Sinaloa. Facultad de Agronomía. Informe final Sistema Nacional de Información sobre Biodiversidad-Comisión Nacional para el Conocimiento y Uso de la Biodiversidad (SNIB-CONABIO) proyecto No. L057. México, D.F., México. 40 pp.

Vega-Aviña, R., H. Aguilar-Hernández, J. A. GutiérrezGarcía, J. A. Hernández-Vizcarra, I. F. Vega-López y J. L. Villaseñor-Ríos. 2000. Endemismo regional presente en la flora del municipio de Culiacán, Sinaloa, México. Acta Botanica Mexicana 53: 1-15. DOI: http://dx.doi. org/10.21829/abm53.2000.858

Vega-Aviña, R., J. L. Villaseñor-Ríos, I. N. Vega-López, J. A Gutiérrez-García, J. A. Hernández-Vizcarra, H. AguiarHernández, G. A. Bojórquez-Bojórquez, J. M. AguilarPatiño y F. Hernández-Álvarez. 2008. Listados Florísticos de Sinaloa I. Flora del municipio de Culiacán, Sinaloa, México. Universidad Autónoma de Sinaloa; Comisión Nacional para el Conocimiento y Uso de la Biodiversidad. Culiacán, México. 81 pp.

Vibrans-Lindemann, H. (ed.). 2011. Malezas de México, Ficha Vigna vexillata (L.) A. Rich. URL: http://www.conabio. gob.mx/malezasdemexico/fabaceae/vignavexillata/ fichas/ficha.htm (consultado marzo de 2017). 
Villaseñor-Ríos, J. L. 2016. Catálogo de las plantas vasculares nativas de México. Revista Mexicana de Biodiversidad 87(3): 559-902. DOI: http://dx.doi.org/10.1016/j. rmb.2016.06.017

Villaseñor-Ríos, J. L. y E. Ortiz-Bermúdez. 2014. Biodiversidad de las plantas con flores (División Magnoliophyta) en México. Revista Mexicana de Biodiversidad 85: 134-142.

DOI: http://dx.doi.org/10.7550/rmb.31987
Wilson, A. K. 1981. Euphorbia heterophylla; a review of distribution, importance and control. Tropical Pest Management 27(1): 32-38. DOI: http://dx.doi. org/10.1080/09670878109414169 